\title{
Enhanced Expression of Hedgehog Pathway Proteins in Oral Epithelial Dysplasia
}

\author{
Rosane Borges Dias, MSc, ${ }^{*}$ Ludmila de Faro Valverde, MSc, ${ }^{*}$ Caroline Brandi Schlaepfer Sales, MSc, \\ Vanessa Sousa Nazaré Guimarães, BSc,* Márcia Grillo Cabral, PhD, † \\ Flávia Caló de Aquino Xavier, PhD, + Jean Nunes dos Santos, PhD, + \\ Eduardo Antônio Gonçalves Ramos, PhD,* and Clarissa Araújo Gurgel Rocha, PhD*
}

\begin{abstract}
The aim of this study was to characterize the profile of the proteins involved in the Hedgehog signaling pathway to aid in the understanding of the pathogenesis of oral epithelial dysplasia (OED). The proteins SHH, PTCH1, HHIP, SUFU, GLI1, and cyclin D1 were evaluated by immunohistochemistry in 25 cases of OED, 4 of non-neoplasic oral mucosa, 8 of inflammatory fibrous hyperplasia and 5 of hyperkeratosis. SHH proteins were predominant in OED cases. Although PTCH1 protein was observed in all cases, this molecule was more highly expressed in OED. The inhibitor protein SUFU was present in OED and HHIP protein was overexpressed in OED. GLI1 proteins were predominantly found in the nuclei of epithelial cells in OED. Basal and suprabasal cells in the epithelial lining were positive for cyclin D1 only in OED. In conclusion, comparative analysis of the proteins involved in the Hedgehog pathway suggests that enhanced expression of these proteins can play an important role in the biological behavior of OED.
\end{abstract}

Key Words: oral epithelial dysplasia, Hedgehog proteins, premalignant lesion

(Appl Immunohistochem Mol Morphol 2016;24:595-602)

$\mathrm{O}$ ral epithelial dysplasias (OED) are potentially malignant lesions whose mechanisms, with respect to pathogenesis and potential neoplastic transformation to

Received for publication February 27, 2015; accepted April 29, 2015.

From the *Laboratory of Pathology and Molecular Biology, Oswaldo Cruz Foundation; \$Laboratory of Oral Surgical Pathology, School of Dentistry of the Federal University of Bahia, Bahia; and $\dagger$ Department of Pathology and Oral Diagnosis, School of Dentistry of the Federal University of Rio de Janeiro, Rio de Janeiro, Brazil.

Supported by the National Council for Scientific and Technological Development (CNPq, Brazil). Foundation to Support Research in the State of Bahia (FAPESB, Bahia, Brazil).

The authors declare no conflicts of interest.

Reprints: Clarissa Araújo Gurgel Rocha, PhD, Oswaldo Cruz Foundation, Gonçalo Moniz Research Center, Waldemar Falcão Street, 121, Candeal-Salvador/BA CEP: 40296-710, Salvador, Bahia, Brazil (e-mails: cgurgel@bahia.fiocruz.br, gurgel.clarissa@gmail.com).

Supplemental Digital Content is available for this article. Direct URL citations appear in the printed text and are provided in the HTML and PDF versions of this article on the journal's Website, www.appliedimmunohist.com.

Copyright (C) 2015 Wolters Kluwer Health, Inc. All rights reserved. oral squamous cell carcinoma (OSCC), are poorly understood. ${ }^{1}$ The Hedgehog $(\mathrm{HH})$ signaling pathway is known to play a key role in the embryonic development of several organs, ${ }^{2}$ such as the teeth,${ }^{3}$ lungs, ${ }^{4}$ colon, breasts, esophagus, ${ }^{5}$ prostate, and stomach, ${ }^{6}$ in addition to participating in the maintenance and growth of adult stem cells, ${ }^{7}$ as well as in tissue repair. ${ }^{8}$ The deregulation of $\mathrm{HH}$ has been associated with the development and progression of some human tumors, including cancers of the prostate, ${ }^{9}$ gastrointestinal tract, ${ }^{10}$ and breast. ${ }^{11,12}$

In humans, this pathway is mainly activated by the Sonic Hedgehog (SHH) ligand, which binds to the patched transmembrane receptors (PTCH1 and PTCH2), resulting in the release of smoothened (SMO) signaling activity. This leads to the activation and nuclear translocation of GLI-Krüppel transcription factors (GLI1, 2, or 3), especially GLI1, causing the transcriptional activation of target genes, such as cyclin D1 (CCND1). ${ }^{6,13}$ Suppressor of fused (SUFU) and hedgehog-interacting protein (HHIP) are negative regulators of the HH pathway. ${ }^{6}$ In the presence of $\mathrm{HH}$ ligands, SUFU dissociates from GLI proteins, thereby promoting their activation and translocation to the nucleus. ${ }^{14}$ In addition, HHIP binds to all $3 \mathrm{Hh}$ proteins with the same affinity and is a direct transcriptional target of $\mathrm{HH}$ signaling. ${ }^{15}$

To the best of our knowledge, the literature contains no reports regarding the participation of this pathway in OED. Recognizing the potential for malignant transformation, and considering that this cascade is reactivated in OSCC, ${ }^{16-18}$ the present study aimed to characterize the profile of the proteins involved in the $\mathrm{HH}$ signaling pathway to aid in the understanding of the pathogenesis of OED.

\section{MATERIALS AND METHODS}

The proteins SHH, PTCH1, HHIP, SUFU, GLI1, and cyclin D1 (CCND1) were evaluated in 25 study subjects with OED at the following sites: buccal mucosa $(\mathrm{n}=8,32 \%)$, tongue $(\mathrm{n}=5,20 \%)$, hard palate $(\mathrm{n}=4$, $16 \%)$, gingival margins $(\mathrm{n}=3,12 \%)$, or mouth floor $(\mathrm{n}=2,8 \%)$. For comparative analysis, the following were also included: 4 cases of non-neoplasic oral mucosa (NNM) obtained from the retromolar gingiva; 8 of inflammatory fibrous hyperplasia (IFH) from the alveolar ridge $(\mathrm{n}=3$, $37,5 \%)$, hard palate $(\mathrm{n}=2,25 \%)$, retromolar gingiva 
$(\mathrm{n}=2,25 \%)$, and buccal mucosa $(\mathrm{n}=1,12.5 \%) ; 5$ cases of hyperkeratosis without dysplastic changes from the tongue $(\mathrm{n}=2,40 \%)$, alveolar ridge $(\mathrm{n}=2,40 \%)$, or gingival margins $(\mathrm{n}=1,20 \%)$. All samples were collected in accordance with the ethical principles established by Gonçalo Moniz Research Center (Oswald Cruz Foundation, Bahia, Brazil) Institutional Review Board.

\section{Histological Examination}

All OEDs were examined by 2 oral pathologists and classified, in accordance with the architectural and cytological criteria established by the World Health Organization (WHO, 2005), ${ }^{19}$ as mild, moderate, or severe. Lesions were also categorized according to perceived risk of malignant transformation using the binary histological grading system proposed by Kujan et al. ${ }^{20}$

\section{Immunohistochemistry}

Sections were obtained at a thickness of $4 \mu \mathrm{m}$, then fixed in formalin and embedded in paraffin. All sections were then submitted to deparaffinization in xylene and subsequently rehydrated with alcohol. Endogenous peroxidase was blocked for 10 minutes under dark conditions (Peroxidase Blocking Solution; Dako, Carpinteria, CA), and tissue proteins were also blocked for 10 minutes (Protein Blocking Solution; Dako). The primary antibodies were incubated for 1 hour at room temperature, with the exception of PTCH1, which was incubated overnight at $4{ }^{\circ} \mathrm{C}$. All data related to the commercial source, clones, and dilutions of antibodies are detailed in Table 1.

Histological sections were then incubated with the HRP Link and HRP Enzyme reagents (Advance; Dako) for 20 minutes each. Reactions were developed using 3, 3'diaminobenzidine and slides were then counterstained with Harris hematoxylin. For negative controls, each antibody was substituted with normal serum of the same isotope as the primary antibody.

\section{Immunohistochemical Analysis}

The location in which positive proteins were detected, for example, membrane, cytoplasm, or nucleus, was recorded, in addition to the location of the lesion, for example, epithelial lining or lamina propria. All histological sections were displayed on an LCD monitor at final magnifications of $\times 200$ and $\times 400$. Images were captured using a Zeiss Axioskop microscope with the aid of an AxioCam HRc camera. All images were analyzed using Image $\mathbf{J}$ software (National Institutes of Health $[\mathrm{NIH}]$, 1997). In each case, positive and negative cells were counted in 5 consecutive microscope fields. By dividing the number of positive cells over the total number of counted cells, protein semiquantification was performed using the following criteria described by Gurgel et $\mathrm{al}^{21}$ : $(-)$ negative, $\leq 5 \%$ of immunostained cells; $(1+)$ Discrete, between $6 \%$ and $25 \%$ of immunostained cells; $(2+)$ moderate, between $26 \%$ and $50 \%$ of immunostained cells; or $(3+)$ intense, $>50 \%$ of immunostained cells.

\section{Statistical Analysis}

All data were submitted to statistical analysis using Mann-Whitney nonparametric testing (GraphPad Prism 6.03; San Diego, CA).

\section{RESULTS}

With respect to WHO classification (2005), ${ }^{19} 21$ $(84 \%)$ cases were classified as mild, $2(8 \%)$ as moderate, and $2(8 \%)$ as severe OED. The distribution of $\mathrm{HH}$ molecules according to WHO criteria is described in Table 2. Based on the binary system proposed by Kujan et al, ${ }^{20} 21(84 \%)$ cases were classified as low-risk OED and $4(16 \%)$ were found to be high risk. The distribution of $\mathrm{HH}$ proteins in these groups can be visualized in Figure 1 in accordance with these scoring criteria.

\section{OED Cases Exhibit Cytoplasmic Positivity for SHH}

Fourteen cases of OED $(56 \%)$ were positive for the SHH protein, with a predominant score of $2+(\mathrm{n}=8$; $57.14 \%)$, followed by a score of $1+(\mathrm{n}=4 ; 28.57 \%)$, and $3+(\mathrm{n}=2 ; 14.29 \%)$. In all cases, the SHH protein was located in the cytoplasm of epithelial cells, most notably in the middle third of the epithelium. All cases of NNM and IFH were found to be negative for the SHH protein, whereas cytoplasmic staining detected just one instance of this protein in a single sample of hyperkeratosis with a score of $1+$ (Figs. 2A-D and Tables 3 and 4).

\section{PTCH1 Expression Was a Common Finding in Cases of OED, NNM, IFH, and Hyperkeratosis}

PTCH1 protein expression was observed in $23(92 \%)$ of the 25 OED cases. In $14(60.87 \%)$ of these, immunostaining was present in the epithelial lining and lamina propria, whereas expression was observed only in epithelial cells in 9 cases $(39.13 \%)$. Cells were scored as $3+$ in the lamina propria in all OED cases, and a score of $3+$ predominated in the epithelial compartment $(\mathrm{n}=17$; $73.91 \%)$, followed by $2+(n=4 ; 17.39 \%)$ and $1+(n=2$; $8.70 \%$ ) (Fig. $2 \mathrm{H}$ ).

In the NNM cases, immunostaining was observed in the membrane, involving cells in the epithelial lining, with

TABLE 1. Data Pertaining to Antibody Clones, Antigen Recovery, and Dilutions

\begin{tabular}{llllc}
\hline Antibody & \multicolumn{1}{c}{ Source } & \multicolumn{1}{c}{ Clone } & \multicolumn{1}{c}{ Positive Control } & \multicolumn{1}{c}{ Antigen Retrieval } \\
\hline SHH & Santa Cruz (SC 9024) & Polyclonal & Placenta & Citrate pH 6 \\
PTCH1 & Novus Biologicals (NBP1-47945) & $5 \mathrm{c} 7$ & Placenta & Without antigen retrieval \\
SUFU & Santa Cruz (SC 28847) & Polyclonal & Placenta & Citrate pH 6 \\
HHIP & Sigma (HPA012616) & Polyclonal & Placenta & Citrate pH 6 \\
GLI1 & Novus Biologicals (NB600-600) & Polyclonal & Placenta & Citrate pH 6 \\
CCND1 & Dako & SP4 & Squamous carcinoma & Citrate pH 6 \\
\hline
\end{tabular}




\begin{tabular}{|c|c|c|c|}
\hline & Mild OED, n (\%) & Moderate OED, n (\%) & Severe OED, n (\%) \\
\hline \multicolumn{4}{|l|}{ SHH } \\
\hline Positive & $13(61.9)$ & $0(0)$ & $1(50)$ \\
\hline Negative & $8(38.1)$ & $2(100)$ & $1(50)$ \\
\hline \multicolumn{4}{|l|}{ PTCH1 } \\
\hline Positive & $20(95.2)$ & $1(50)$ & $2(100)$ \\
\hline Negative & $1(4.8)$ & $1(50)$ & $0(0)$ \\
\hline \multicolumn{4}{|l|}{ SUFU } \\
\hline Positive & $8(38.1)$ & $0(0)$ & $2(100)$ \\
\hline Negative & $13(61.9)$ & $2(100)$ & $0(0)$ \\
\hline \multicolumn{4}{|l|}{ HHIP } \\
\hline Positive & $15(71.4)$ & $2(100)$ & $0(0)$ \\
\hline Negative & $6(28.6)$ & $0(0)$ & $2(100)$ \\
\hline \multicolumn{4}{|l|}{ GLI1 } \\
\hline Positive & $17(81)$ & $2(100)$ & $2(100)$ \\
\hline Negative & $4(19)$ & $0(0)$ & $0(0)$ \\
\hline \multicolumn{4}{|l|}{ CCND1 } \\
\hline Positive & $16(76.2)$ & $2(100)$ & $2(100)$ \\
\hline Negative & $5(23.8)$ & $0(0)$ & $0(0)$ \\
\hline
\end{tabular}

OED indicates oral epithelial dysplasia.

scores of $1+(\mathrm{n}=2 ; 50 \%)$ and $2+(\mathrm{n}=2,50 \%)$ (Fig. 2E). Positivity was also observed in the lamina propria. Similarly, PTCH1 was detected in the epithelial compartment of all hyperkeratosis cases, with scores ranging from $2+(\mathrm{n}=3,60 \%)$ to $3+(\mathrm{n}=2,40 \%)$ (Fig. 2G). Immunopositivity was also detected in the lamina propria in $4(80 \%)$ of these cases.

PTCH1 protein expression was present in most of the IFH cases $(n=5,62.5 \%)$, but exclusively located in the membranes of epithelial cells (score $1+: \mathrm{n}=2,40 \%$; score $2+: n=2,40 \%$; score $3+: n=1,20 \%$ ) (Fig. $2 F)$. Immunostaining score distribution for PTCH1 and corresponding statistical analysis are presented in Tables 3 and 4 , respectively.
The Inhibitor Protein SUFU Was Present in OED, But Not in NNM

SUFU protein was positive in the cytoplasm of cells in the epithelial lining in $11(44 \%)$ OED cases, with 4 $(36.36 \%)$ of these scored as $3+, 4(36.36 \%)$ as $2+$ and 3 $(27.28 \%)$ as $1+$. Although no SUFU expression was detected in the NNM cases, positive cells were detected in one case of hyperkeratosis and in an instance of IFH, both receiving a score of $1+$ (Figs. 2I-L and Tables 3 and 4).

\section{HHIP Is Overexpressed in OED, But Not in NNM, IFH, and Hyperkeratosis}

Seventeen $(68 \%)$ of the OED cases were positive for HHIP protein, which was observed in the membrane of

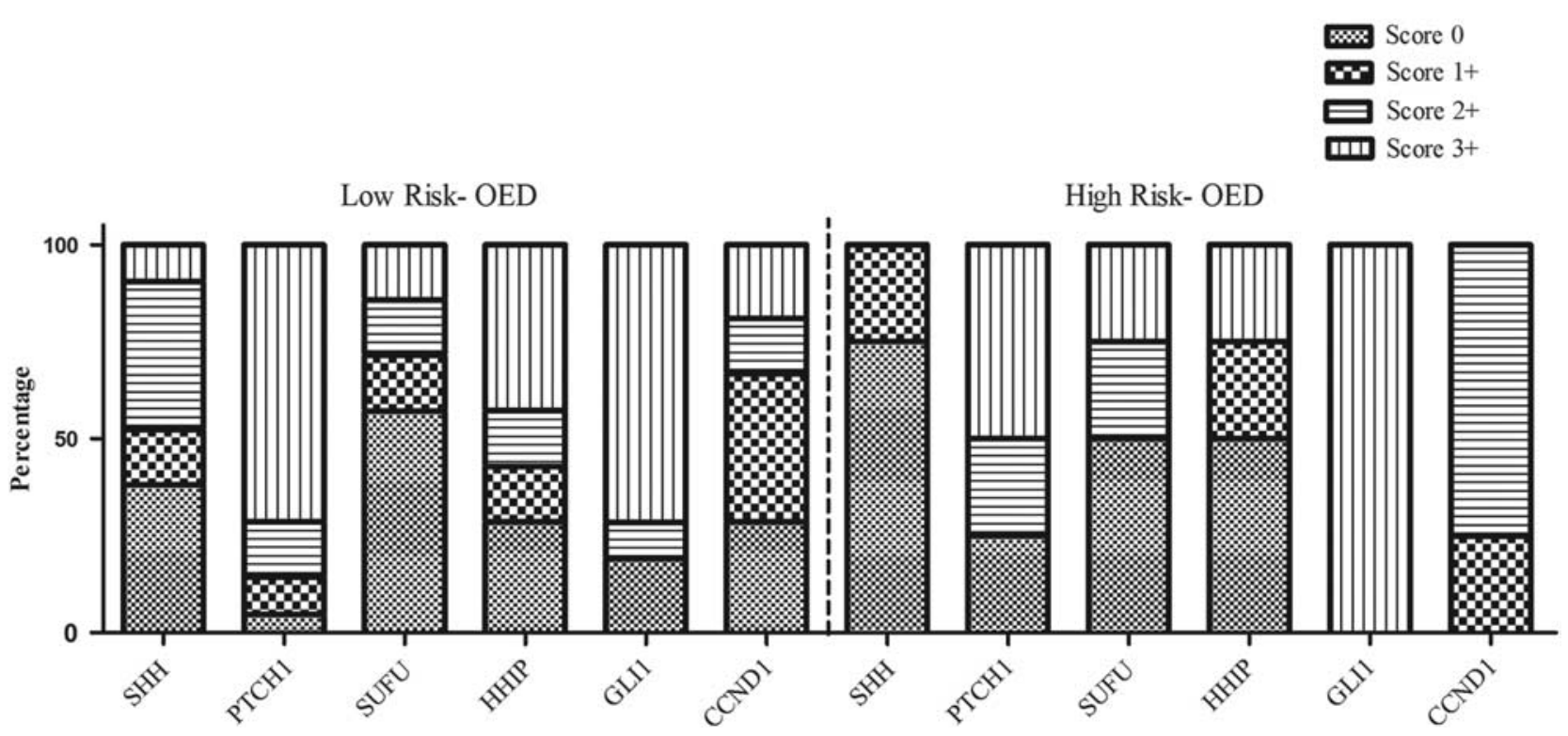

FIGURE 1. Expression distributions of Hedgehog proteins in OED according to binary system proposed by Kujan et al. ${ }^{20}$ 
TABLE 3. Immunostaining Score for OED, Hyperkeratosis, IFH, and NNM

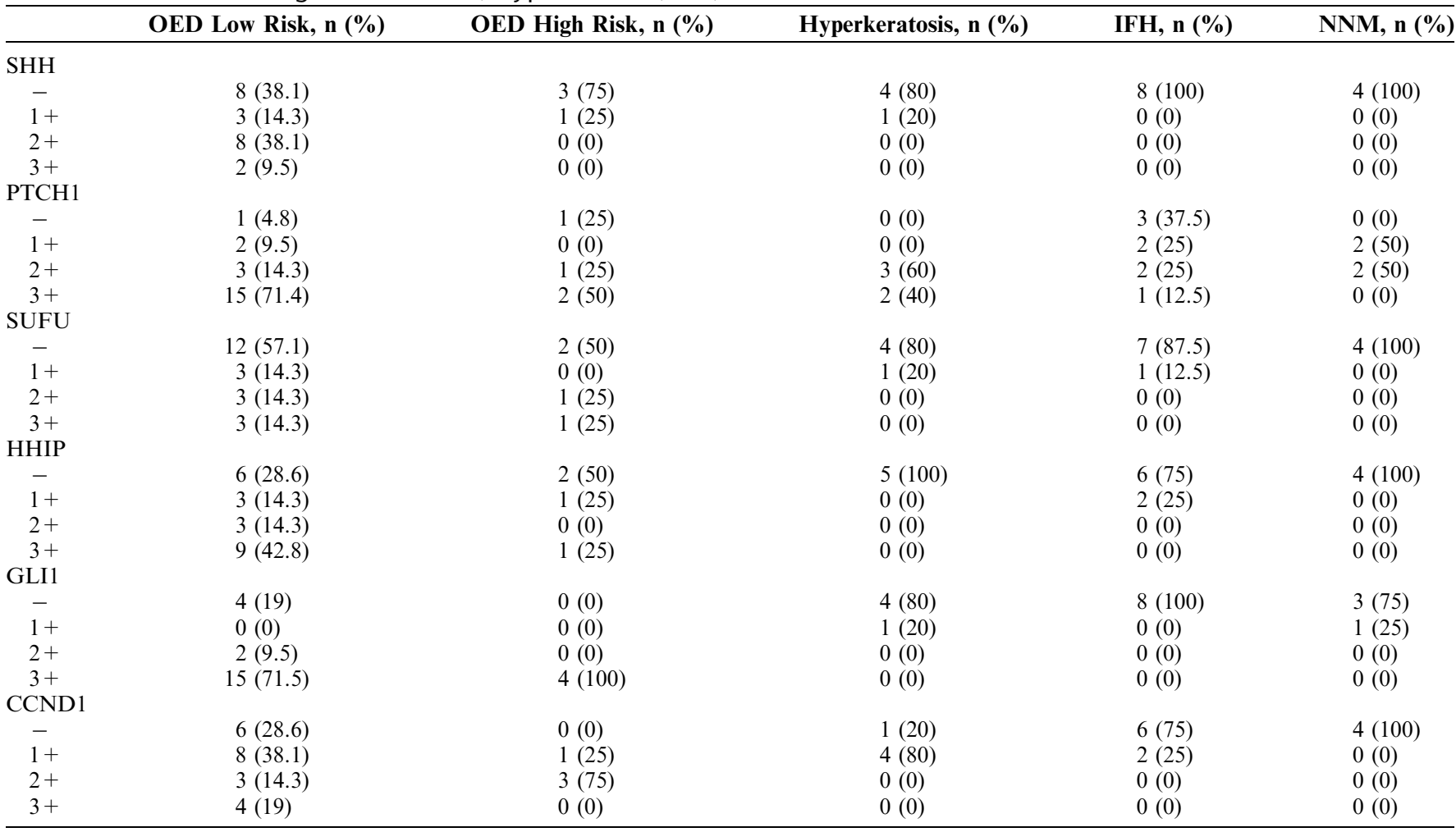

IFH indicates inflammatory fibrous hyperplasia; NNM, non-neoplasic oral mucosa; OED, oral epithelial dysplasia

epithelial cells, with a predominant score of $3+(\mathrm{n}=10$, $58.82 \%)$, followed by $1+(\mathrm{n}=4,23.53 \%)$ and $2+(\mathrm{n}=3$, $17.65 \%$ ). The NNM and hyperkeratosis samples were all negative for HHIP, whereas 2 cases of IFH exhibited cytoplasmic staining scores of $1+$ (Figs. $2 \mathrm{M}-\mathrm{P}$ and Tables 3 and 4).

TABLE 4. Comparison of Hedgehog Proteins in OED, NNM, Hyperkeratosis, and IFH

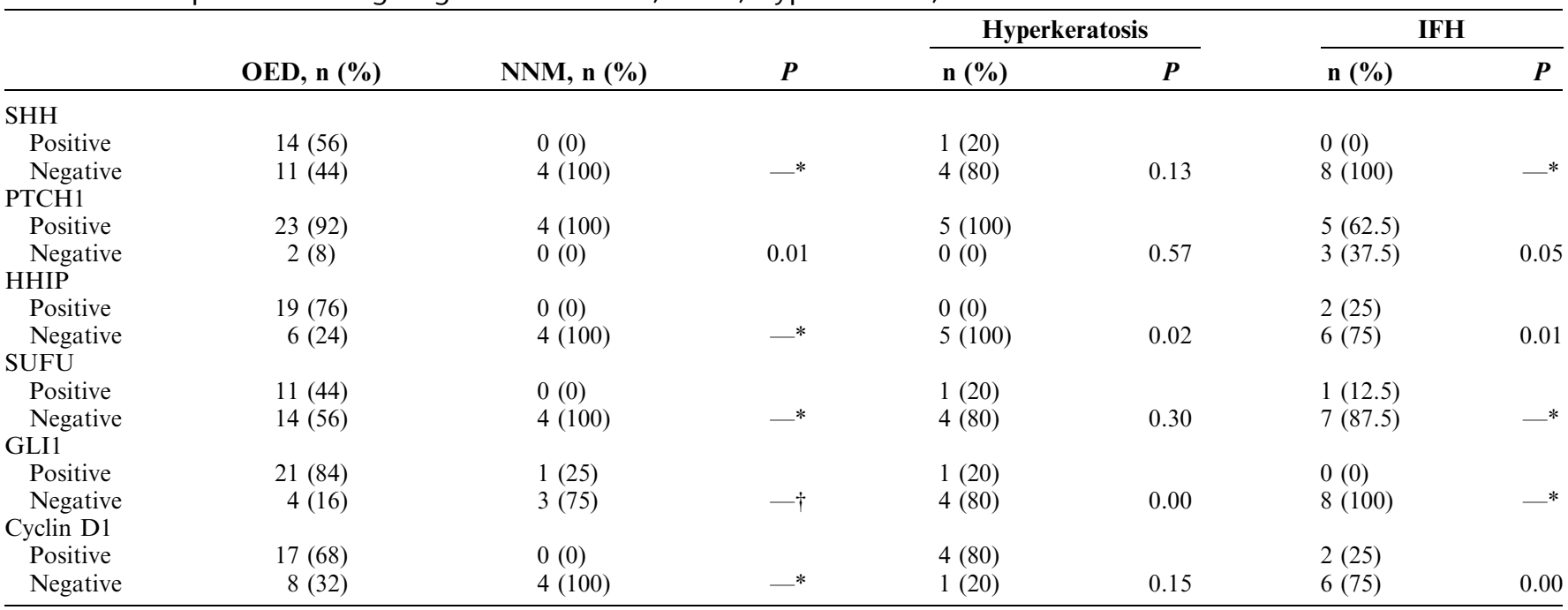

*Statistical analyses were not performed: IFH and/or NNM with immunostaining values equal to zero. HOED not compared with NNM: GLI1 detected in the cytoplasm of normal cells.

IFH indicates inflammatory fibrous hyperplasia; NNM, non-neoplasic oral mucosa; OED, oral epithelial dysplasia. 


\section{GLI1 Was Predominantly Found in the Nuclei of Epithelial Cells in OED}

The GLI1 protein was positive in $21(84 \%)$ OED cases, with a predominant score of $3+(\mathrm{n}=19,90.48 \%)$, whereas the remaining $2(9.52 \%)$ cases received a score of $2+$. GLI1 was observed exclusively in the nucleus in 4 $(19.05 \%)$ cases, and in the nucleus and cytoplasm in 17 $(80.95 \%)$. One NNM and a single hyperkeratosis case showed positivity for GLI1 in the cytoplasm, both scored as $1+$, and all IFH cases were negative for this protein (Figs. 2Q-T and Tables 3 and 4).

\section{Basal and Suprabasal Cells in the Epithelial Lining of OED Cases Were Positive for CCND1}

Nineteen $(76 \%)$ OED cases were positive for the CCND1 protein, with $9(47.37 \%)$ presenting immunostaining scored as $1+, 6(31.58 \%)$ as $2+$, and 4 $(21.05 \%)$ as $3+$. This protein was exclusively found in the nuclei of epithelial lining cells. All NNM samples were negative, whereas $4(80 \%)$ hyperkeratosis samples and 2 $(25 \%)$ IFH cases were positive for CCND1, both presenting a score of $1+$ (Figs. $2 \mathrm{U}-\mathrm{X}$ and Tables 3 and 4 ).

\section{DISCUSSION}

The present study compared the protein expression found in the HH pathway in 25 cases of OED with that observed in histologically normal tissue from NNM, as well as reactive lesions from hyperkeratosis and IFH.

In this study, all samples (OED, NMM, IFH, and hyperkeratosis) were analyzed by 2 experienced pathologists. At the beginning of our study, a total of 48 OED were selected and 23 cases were excluded in accordance with one exclusion criterion: atypical cells related to inflammation.

Histological grading indicated a predominance of mild lesions $(\mathrm{n}=21,84 \%)$ in the OED cases according to WHO criteria, and the risk of malignant transformation was determined to be low. Although no histological classification system exists that can accurately predict which determining factors lead OED to progress to OSCC, there is a consensus among pathologists that the binary system proposed by Kujan et $\mathrm{a}^{20}$ is the best prognostic indicator currently available with respect to risk of malignant transformation. ${ }^{22}$

The signaling pathways involved in embryonic development are normally maintained in an inactive state in nonneoplastic adult tissues, and it is known that reactivation may lead to tumor development. ${ }^{6,7}$ Recently published evidence indicates that the $\mathrm{HH}$ pathway appears to play an important role in the pathogenesis of OSCC ${ }^{16-18,23}$; however, to the best of our knowledge, no studies have investigated this signaling pathway in any cases of OED.

As the present results demonstrate the activation of the HH pathway in OED, we hypothesize that the proteins involved in this signaling pathway may be associated with the initial stages of carcinogenesis, that is at an earlier stage of oral cancer development; as such, these should be considered as potential candidates for biomarkers of this disease.
When activated, the HH pathway plays an important role in the transcription of genes related to cellular proliferation and differentiation, such as in cyclin D1 and $\beta$-catenin. ${ }^{24}$ The participation of this pathway in malignant tumors has already been well established. ${ }^{25-29}$ In addition, there is evidence of the involvement of $\mathrm{HH}$ pathway molecules in precursor lesions of the colon, ${ }^{30}$ uterine cervix, ${ }^{31}$ and pancreas. ${ }^{27}$

This pathway can be reactivated via a variety of mechanisms, with or without participation of the SHH ligand, through the mutational activation of SMO protein or by mutations in PTCH1.32 The presence of SHH morphogens found exclusively in the cytoplasm of epithelial cells obtained from the middle third in $14(56 \%)$ cases of OED indicates that this ligand may participate in the autocrine activation of the $\mathrm{HH}$ pathway during the course of this disease. In other precursor lesions, such as those in the colon and cervix, SHH ligand has also been detected in the cytoplasm of genetically altered cells with greater expression than in non-neoplastic tissues. ${ }^{30,31}$

In the present study, PTCH1 was observed in the membranes of epithelial cells and in the lamina propria in all lesion types considered, whereas immunopositivity was markedly more intense in OED cases, notably involving greater numbers of cells. Constitutive expression of this protein is an expected finding in adult tissues, as it acts as a tumor suppressor protein ${ }^{25}$ and negative regulator of the HH pathway. ${ }^{6}$ Although lower levels of PTCH1 expression are to be expected in non-neoplastic tissues, ${ }^{30,31}$ overexpression is commonly observed in malignant neoplasia, ${ }^{4,33}$ which explains why the PTCH1 gene is targeted by GLI1, and why the detection of high levels of this protein are indicative of $\mathrm{HH}$ signaling pathway activation. ${ }^{29}$

Similarly, increased expression of SUFU and HHIP is also to be expected when $\mathrm{HH}$ is activated. Both proteins act as tumor suppressors and are encoded by genes targeted by this cascade. ${ }^{6}$ While $17(68 \%)$ and $11(44 \%)$ OED samples exhibited positivity for HHIP and SUFU, respectively, all NNM samples were negative for both of these proteins. In the reactive lesion samples, 2 cases of IFH and 1 of hyperkeratosis were found to be positive for both proteins. Even though the detection of PTCH1, HHIP and SUFU contribute to the activation of the $\mathrm{HH}$ pathway in OED cases, these proteins are also capable of suppressing malignancy events. 15,34

One of the most remarkable findings reported herein was that nuclear staining of GLI1 was observed in 21 OED cases. This finding serves as confirmation of participation by the HH pathway in OED, since the nuclear translocation of this protein occurs only after activation of this signaling cascade. ${ }^{35}$ Interestingly, this pattern of immunostaining was not observed in any cases of NNM, hyperkeratosis or IFH. Since the nuclear expression of GLI1 is related to a very unfavorable prognosis in several types of malignant tumors, ${ }^{35}$ this finding urges the evaluation of GLI1 as a biomarker for OED in the context of a larger case series. 

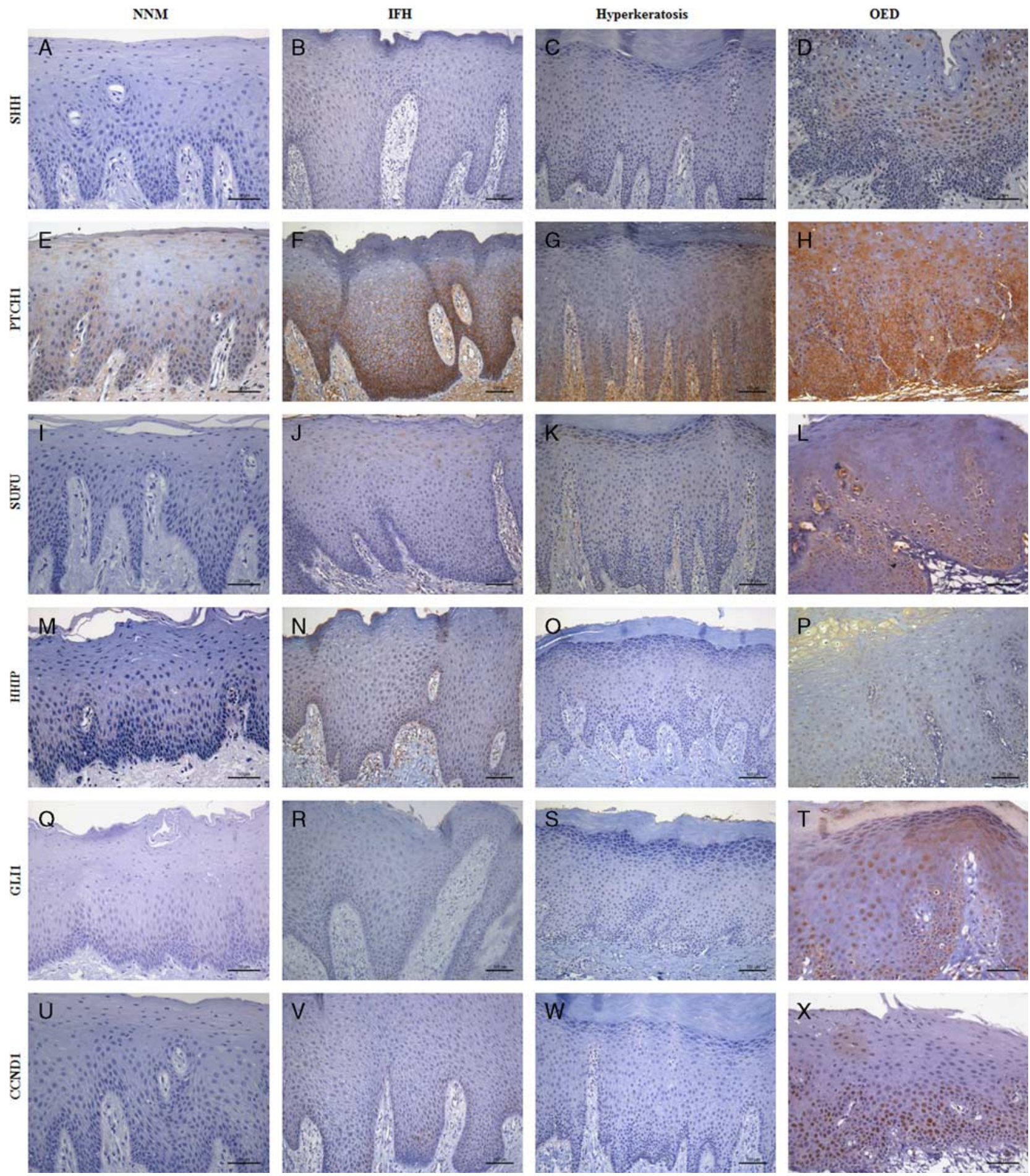

FIGURE 2. (A-D)-SHH: (A-C) Negative scores for non-neoplastic oral mucosa (NNM), inflammatory fibrous hyperplasia (IFH) and hyperkeratosis. (D) Moderate/high risk oral epithelial dysplasia (OED) with a score of 3+. (E-H)-PTCH1: (E and G). Discrete immunostaining and membrane pattern in NNM and hyperkeratosis. (F) Intense immunostaining of the membrane in IFH (H) Predominant membrane pattern of severe/high risk OED. (I-L)-SUFU: (I and K) Negative epithelial cells in samples of NNM. and hyperkeratosis. (J) Cytoplasmic immunostaining in IFH with a score of $1+$. (L) Discrete/low risk OED with a cytoplasmic staining score of 2+. (M-P)-HHIP: (M, N) Negative epithelial cells in samples of NNM, and hyperkeratosis. (O) Cytoplasmic immunostaining in IFH with score ++ . (P) Cytoplasmic and perinuclear immunostaining in discrete/low risk oral epithelial dysplasia (OED) with a score of 3+. (Q-T)- GLI1: (Q-S). Negative scores for NNM, IFH and hyperkeratosis. (T) Predominant nuclear pattern in discrete/low risk OED with a score of 3+. (U-X)-CCND1: (U and W) Negative scores for NNM and hyperkeratosis. ( $\mathrm{V}$ and $\mathrm{X}$ ) Nuclear immunostaining in IFH and discrete/low risk OED with a score of $1+$. 
Despite the fact that CCND1 expression can be regulated by a variety of mechanisms, ${ }^{36}$ one effect of the nuclear translocation of GLI1 is the overexpression of this protein. ${ }^{37}$

It is important to note that the detection of CCND1 in OED has been described in both basal as well as suprabasal layers of the epithelium. ${ }^{38,39}$ Our results corroborate this evidence and also demonstrate that these lesions exhibit a suprabasal proliferative compartment. By contrast, the included NNM samples were considered to be negative as they showed low immunopositivity $(<5 \%)$ for this protein, which is consistent with reports by other authors who also used NNM for comparative purposes. ${ }^{38}$ In the hyperkeratosis and IFH cases, $4(80 \%)$ and $2(25 \%)$ samples, respectively, demonstrated positivity in cells located in the basal and parabasal epithelial compartments. Nonetheless, CCND1 expression should be considered as a possible result of inflammation in these cases, as was previously demonstrated by Nair et al. ${ }^{40}$

In conclusion, comparative analysis of the proteins involved in the HH pathway in the present OED, NNM, IFH, and hyperkeratosis cases strongly suggests that this pathway plays an important role in the biological behavior of OED. Notably, when compared with IFH, the OED samples exhibited higher levels of expression with respect to all the $\mathrm{HH}$ molecules involved, substantiating the notion that these proteins may influence carcinogenesis, regardless of the associated inflammatory process. Our results additionally provide convincing evidence that this signaling cascade becomes reactivated in OED lesions. Moreover, the expression profiles of SHH, PTCH1, and HHIP genes further corroborate this evidence (Fig. S1, Supplemental Digital Content, http:// links.lww.com/AIMM/A81). To evaluate whether the molecules modulated by this pathway, such as the GLI1 protein, could represent relevant biomarkers of this disease, it is necessary to widen the scope of study using a more representative sampling, in addition to proficiently observing patients to identify which OED cases actually progress to squamous cell carcinoma. Alternatively, evaluating expression levels of the genes and proteins involved in the $\mathrm{HH}$ pathway using an experimental carcinoma model at different stages of carcinogenesis may further the understanding of how these molecules participate in tumor pathogenesis.

\section{ACKNOWLEDGMENT}

The authors thank Andris $K$. Walter for providing English translation and consulting services.

\section{REFERENCES}

1. Wang Z, Feng X, Liu X, et al. Involvement of potential pathways in malignant transformation from oral leukoplakia to oral squamous cell carcinoma revealed by proteomic analysis. BMC Genomics. 2009; 10:383.

2. Hassounah NB, Bunch TA, McDermott KM. Molecular pathways: the role of primary cilia in cancer progression and therapeutics with a focus on Hedgehog signaling. Clin Cancer Res. 2012;18:2429-2435.

3. Hardcastle Z, Mo R, Hui C, et al. The Shh signalling pathway in tooth development: defects in Gli2 and Gli3 mutants. Development. 1998;125:2803-2811.
4. Grindley JC, Perkins D, Hogan BLM. Evidence for the involvement of the Gli gene family in embryonic mouse lung development. Dev Biol. 1997;188:337-348.

5. Ingham PW, McMahon AP. Hedgehog signaling in animal development: paradigms and principles. Genes Dev. 2001;15:3059-3087.

6. Chari NS, McDonnell TJ. The sonic hedgehog signaling network in development and neoplasia. Adv Anat Pathol. 2007;14:344-352.

7. Ruiz i Altaba A, Sánchez P, Dahmane N. Gli and hedgehog in cancer: tumours, embryos and stem cells. Nat Rev Cancer. 2002;2: 361-372.

8. Asai J, Takenaka H, Kusano KF, et al. Topical sonic hedgehog gene therapy accelerates wound healing in diabetes by enhancing endothelial progenitor cell-mediated microvascular remodeling. Circulation. 2006;113:2413-2424.

9. Karhadkar SS, Bova GS, Abdallah N, et al. Hedgehog signalling in prostate regeneration, neoplasia and metastasis. Nature. 2004;431:707-712.

10. Berman DM, Karhadkar SS, Maitra A, et al. Widespread requirement for Hedgehog ligand stimulation in growth of digestive tract tumours. Nature. 2003;425:846-851.

11. Katano M. Hedgehog signaling pathway as a therapeutic target in breast cancer. Cancer Lett. 2005;227:99-104.

12. Mukherjee S, Frolova N, Sadlonova A, et al. Hedgehog signaling and response to cyclopamine differ in epithelial and stromal cells in benign breast and breast cancer. Cancer Biol Ther. 2006;5:674-683.

13. Scales SJ, de Sauvage FJ. Mechanisms of Hedgehog pathway activation in cancer and implications for therapy. Trends Pharmacol Sci. 2009;30:303-312.

14. Humke EW, Dorn KV, Milenkovic L, et al. The output of Hedgehog signaling is controlled by the dynamic association between suppressor of fused and the Gli proteins. Genes Dev. 2010;24:670-682.

15. Song Y, Tian Y, Zuo Y, et al. Altered expression of PTCH and HHIP in gastric cancer through their gene promoter methylation: novel targets for gastric cancer. Mol Med Rep. 2013;7:1159-1168.

16. Cavicchioli Buim ME, Gurgel CAS, Gonçalves Ramos EA, et al. Activation of sonic hedgehog signaling in oral squamous cell carcinomas: a preliminary study. Hum Pathol. 2011;42:1484-1490.

17. Leovic D, Sabol M, Ozretic P, et al. Hh-Gli signaling pathway activity in oral and oropharyngeal squamous cell carcinoma. Head Neck. 2012;34:104-112.

18. Schneider S, Thurnher D, Kloimstein P, et al. Expression of the Sonic hedgehog pathway in squamous cell carcinoma of the skin and the mucosa of the head and neck. Head Neck. 2011;33:244-250.

19. Gale N, Pilch BZ, Sidransky D, et al. Tumours of the hypopharynx, larynx and trachea (Epithelial precursor lesions). In: Barnes L, Eveson JW, Reichart P, Sidransky D, eds. World Health Organization Classification of Tumours Pathology \& Genetics Head and neck tumours. Lyon: International Agency for Research on Cancer (IARC) Press; 2005:140-143.

20. Kujan O, Oliver RJ, Khattab A, et al. Evaluation of a new binary system of grading oral epithelial dysplasia for prediction of malignant transformation. Oral Oncol. 2006;42:987-993.

21. Gurgel CAS, Ramos EAG, Azevedo RA, et al. Expression of Ki-67, p53 and p63 proteins in keratocyst odontogenic tumours: an immunohistochemical study. J Mol Histol. 2008;39:311-316.

22. Nankivell $\mathrm{P}$, Williams $\mathrm{H}$, Matthews $\mathrm{P}$, et al. The binary oral dysplasia grading system: validity testing and suggested improvement. Oral Surg Oral Med Oral Pathol Oral Radiol. 2013;115:87-94.

23. Honami T, Shimo T, Okui T, et al. Sonic hedgehog signaling promotes growth of oral squamous cell carcinoma cells associated with bone destruction. Oral Oncol. 2012;48:49-55.

24. Katoh Y, Katoh M. Hedgehog target genes: mechanisms of carcinogenesis induced by aberrant Hedgehog signaling activation. Curr Mol Med. 2009;9:873-886.

25. Sicklick JK, Li Y-X, Jayaraman A, et al. Dysregulation of the Hedgehog pathway in human hepatocarcinogenesis. Carcinogenesis. 2006;27:748-757.

26. Che L, Yuan Y-H, Jia J, et al. Activation of sonic hedgehog signaling pathway is an independent potential prognosis predictor in human hepatocellular carcinoma patients. Chin $J$ Cancer Res. 2012;24:323-331. 
27. Thayer SP, di Magliano MP, Heiser PW, et al. Hedgehog is an early and late mediator of pancreatic cancer tumorigenesis. Nature. 2003;425:851-856.

28. Johnson RL, Rothman AL, Xie J, et al. Human Homolog of patched, a candidate gene for the basal cell nevus syndrome. Science. 1996;272:1668-1671.

29. Xie J, Murone M, Luoh S, et al. Activating Smoothened mutations in sporadic basal-cell carcinoma. Nature. 1998;391:90-92.

30. Oniscu A, James RM, Morris RG, et al. Expression of sonic hedgehog pathway genes is altered in colonic neoplasia. J Pathol. 2004;203:909-917.

31. Xuan YH, Jung HS, Choi Y-L, et al. Enhanced expression of hedgehog signaling molecules in squamous cell carcinoma of uterine cervix and its precursor lesions. Mod Pathol. 2006;19:1139-1147.

32. Walterhouse D, Yoon J, Philip M. Developmental pathways: sonic Hedgehog-patched-GLI. Environ Health Perspect. 1999;107:167-171.

33. Yoshikawa K, Shimada M, Miyamoto H, et al. Sonic hedgehog relates to colorectal carcinogenesis. J Gastroenterol. 2009;44:1113-1117.

34. Katoh Y, Katoh M. Hedgehog signaling pathway and gastrointestinal stem cell signaling network (Review). Int J Mol Med. 2006;18:1019-1023.
35. Souzaki M, Kubo M, Kai M, et al. Hedgehog signaling pathway mediates the progression of non-invasive breast cancer to invasive breast cancer. Cancer Sci. 2011;102:373-381.

36. Russell A, Thompson MA, Hendley J, et al. Cyclin D1 and D3 associate with the SCF complex and are coordinately elevated in breast cancer. Oncogene. 1999;18:1983-1991.

37. Bermudez O, Hennen E, Koch I, et al. Glil mediates lung cancer cell proliferation and Sonic Hedgehog-dependent mesenchymal cell activation. PLoS One. 2013;8:e63226.

38. Kövesi G, Szende B. Prognostic value of cyclin D1, p27, and p63 in oral leukoplakia. J Oral Pathol Med. 2006;35:274-277.

39. Ramasubramanian A, Ramani P, Sherlin HJ, et al. Immunohistochemical evaluation of oral epithelial dysplasia using cyclin-D1, p27 and p63 expression as predictors of malignant transformation. J Nat Sci Biol Med. 2013;4:349-358.

40. Nair S, Nguyen H, Salama S, et al. Obesity and the endometrium: adipocyte-secreted proinflammatory TNF cytokine enhances the proliferation of human endometrial glandular cells. Obstet Gynecol Int. 2013;2013:368543. 